\title{
Formation Mechanism of Strip Defects in Anodizing AA5252 Aluminium Alloy Sheets
}

\author{
Chong Gao ${ }^{1,2}$, Zhongyu Jiang $^{3}$, Zhenshan Liu $^{1,2, *}$, Pizhi Zhao $^{1,2,{ }^{*}}$, Ruiyin Huang ${ }^{3}$, Yongfu Wu ${ }^{1,2}$ and Kangcai Yu ${ }^{1,2}$ \\ ${ }^{1}$ Chinalco Materials Application Research Institute Co. Ltd., Institute of light alloy materials, Beijing 102209, China \\ ${ }^{2}$ Chinalco Research Institute of Science and Technology Co. Ltd., Beijing 102209, China \\ ${ }^{3}$ Chinalco Ruimin Co. Ltd., Fuzhou 350015, China
}

\begin{abstract}
Processed by sulfuric acid anodizing, several unexpected strip defects exhibited on the anodizing film of AA5252 aluminium alloy sheets. Their formation mechanism was studied in detail. The normal zone and strip zone were compared, with respect to surface brightness, porosity of anodizing film, and microstructures of the corresponding aluminium substrate tested by spectroscopic colorimeter and SEM, and SEM-EBSD. Results showed that the brightness of strip zone was lower than that of normal zone. Additionally, compared with normal zone, the porosity of anodizing film was higher, and cubic grains fewer, which located in the corresponding surface layer of aluminium substrate in strip zone. In order to further clarify the effect of grain orientation on the brightness of anodizing film, single crystal aluminium sheet with (100), (110) or (111) orientation was anodized. Results showed that the anodized (100) oriented specimen, i.e., cubic grain was brighter, whose anodizing film had lower porosity, compared with (110) and (111) oriented ones. Therefore, the formation mechanism of strip defects was proposed as uneven distribution of cubic grains. The zones with fewer cubic orientated grains were darker, and were visible as strip defects after anodizing.
\end{abstract}

\section{Introduction}

Aluminium and its alloys have attractive attention because of their low density, high strength, good corrosion resistance and electrical conductivity etc. Meanwhile, aluminium alloy materials processed by colour anodizing could present a variety of colours and excellent corrosion protection, they are widely utilized as exterior structural components in $3 \mathrm{C}$ devices, including computer, cell phone and consumer electronic. The surface appearance is a critical characteristic for these components. Unfortunately, strip defects often become visible after anodizing, rather than in the rolled condition. Thus, it difficult to identify the original causes of the defects. To date, to achieve reproducible and wellcontrolled surface appearance of anodized aluminium alloy is still a challenging issue.

Strip defect is typically characterized by narrow bands with a different brightness from the surrounding material, usually darker than the normal zone. The anodizing film formed in sulfuric acid solution consists of a thin compact barrier layer and a thick layer consisting of hexagonally shaped cells. The barrier layer is adjacent to the aluminium substrate, whose thickness is about 1.1 $\mathrm{nm} / \mathrm{V}$ with respect to the applied voltage. And in the other layer the hexagonal cell is a central pore perpendicular to the substrate surface ${ }^{[1,2]}$.

Previous researches ${ }^{[3-5]}$ indicated that the strip defects not only correlate strongly with the anodizing conditions (voltage, temperature, composition and acid concentration of the bath), but also depend on the microstructure of the aluminium substrate, i.e. impurity distribution, grain size, texture and surface morphology. Y. Ma et al. ${ }^{[6]}$ suggested that $\mathrm{Mg}_{2} \mathrm{Si}$ phase in AA6063 aluminium alloy was preferentially oxidized at low anodizing voltages, and resulted in the development of a rough alloy/film interface and the incorporation of silicon into the porous anodic film, which led to the strip defects. H.L. Zhu et al. ${ }^{[7]}$ found that the formation of a heterogeneous distribution of grain boundary grooves is a critical factor for strip defects. The grooves are mainly influenced by the distribution of $\mathrm{Mg}_{2} \mathrm{Si}$ precipitates and Fe-rich intermetallic particles on the surface of 6060 aluminium alloy sheets.

In this study, a high purity AA5252 aluminium alloy sheet was selected as the research target, because the effect of secondary phases, like $\mathrm{Mg}_{2} \mathrm{Si}$ precipitates and Fe-rich intermetallic particles, could be excluded in this investigation. Therefore, the study on the effect of strip defects focused on the grain structure in the anodized AA5252 aluminium alloy sheets. The correlation among the strip defect, the porosity of anodizing film, and the different grain structures was systematically studied, and the formation mechanism of strip defects was discussed.

\section{Experimental procedure}

\subsection{Materials preparation}

Materials for the present study were AA 5252 aluminium alloy sheets with gauge of $0.6 \mathrm{~mm}$ and $\mathrm{H} 22$ temper. The composition of the alloy is given in Table 1 . They were trial-produced on industrial scale, AA5252 alloy ingots were manufactured by direct chill (DC) casting and subjected to homogenization, and subsequently rolled to necessary gauge by hot rolling and cold rolling. These sheets were blasted and subsequently anodized in 20 wt.\% sulfuric acid at $25{ }^{\circ} \mathrm{C}$ for 25 minutes using direct current (DC) with $1.4 \mathrm{~A} \mathrm{dm}^{-2}$, then immersed in blue dye solution, used to manufacture backplane parts of cell phone in batch commercially. Specimens as large as 80 $\mathrm{mm} \times 50 \mathrm{~mm}$ was cut by electrical discharge machining

\footnotetext{
* Corresponding author: zhaopizhi@,cmari.com, liuzhenshan@,cmari.com
} 
from the area without plastic deformation in the backplane.

Table 1. Chemical composition of AA5252 aluminium alloy sheets (wt./\%).

\begin{tabular}{ccccccc}
\hline $\mathrm{Si}$ & $\mathrm{Fe}$ & $\mathrm{Cu}$ & $\mathrm{Mn}$ & $\mathrm{Mg}$ & $\mathrm{Zn}$ & $\mathrm{Bal}$ \\
\hline$\leq 0.02$ & $\leq 0.04$ & $\leq 0.10$ & $\leq 0.10$ & $2.2 \sim 2.8$ & $\leq 0.05$ & $\mathrm{Al}$ \\
\hline
\end{tabular}

Furthermore, (100), (110) and (111) orientated single crystals of pure aluminium ( $\mathrm{Al} \geq 99.99$ wt.\%) were prepared through Bridgeman method, to understand the effect of grain orientations on the anodic films properties. Square specimens with the size of $10 \mathrm{~mm} \times 10 \mathrm{~mm}$ were prepared for the investigation. The orientations of the samples were verified by X-ray diffraction (XRD), as shown in Fig.1. Before the anodization, specimens were mirror polished. They were anodized in $20 \mathrm{wt} . \%$ sulfuric acid at $25{ }^{\circ} \mathrm{C}$ for 25 minutes using direct current (DC) with $1.4 \mathrm{~A} \mathrm{dm}^{-2}$, subsequently sealed in boiled water and dried in air.

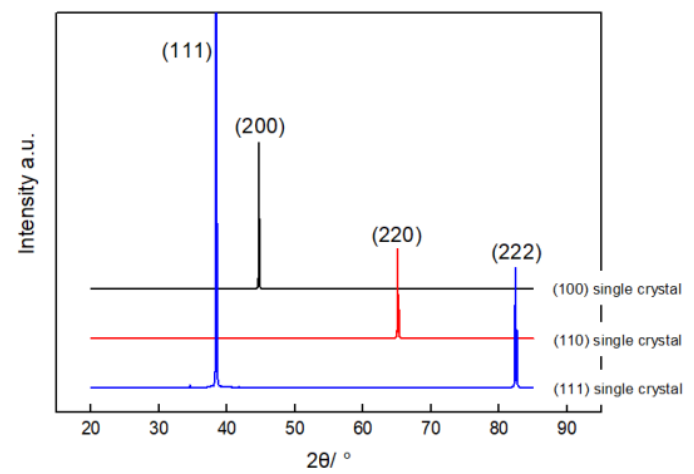

Fig. 1. XRD spectrum of single crystal aluminium alloys.

\subsection{Microstructure analysis}

The appearance of anodized specimens was recorded in D65 light source with a viewing angle of about $45^{\circ}$. The surface brightness $\mathrm{L}^{*}$ of the anodized aluminium was measured by a 3NH YS3060 high-precision spectrophotometer. The value $\mathrm{L}^{*}$ ranges from 0 to 100 represent for black-and-white. The porosity of anodizing film refers to the percentage of pore area in the observed total area. It was investigated quantitatively by a Hitachi SU8010 ultra high-resolution field emission scanning electron microscope (SEM) from the top view. The thickness of anodizing film was measured quantitatively by a Fischer FMP 40 equipped with a FTA3.3H probe. To reveal the microstructure on the surface of aluminium substrate, the anodized AA5252 aluminium alloy specimen cut from cell phone backplane was ground 1 min with sandpaper (1200\#), and then mirror polished for $3 \mathrm{~min}$ to remove the anodizing film. The specimen thickness reduced around $10 \sim 15 \mu \mathrm{m}$, which is slightly over the thickness of anodizing film. For comparison, secondary phases and grain structures of AA5252 aluminium alloy sheets both located under the strip defects and the normal zone were evaluated respectively by a JEOL JSM-7800F SEM equipped with Electron Backscatter Diffraction (EBSD).

\section{Results and discussion}

\subsection{Strip defects}

Fig. 2 presented a typical anodized AA5252 aluminium alloy part of cell phone. The thickness of anodizing film was $8.9 \pm 0.4 \mu \mathrm{m}$. Several strip defects appeared on the surface. Strip defects showed strip patterns with random distribution along the rolling direction. Strip zones were dark, their width less than $1.0 \mathrm{~mm}$, length up to tens of millimetres. The brightness $\mathrm{L}^{*}$ of the normal zone and strip zone was $87.93 \pm 0.11$ and $87.59 \pm 0.10$ respectively, the deviation $\triangle L^{*}=0.34$. This indicated that the strip zone was darker than normal zones, and was visual slightly on the appearance of anodized AA5252 aluminium alloy sheets.

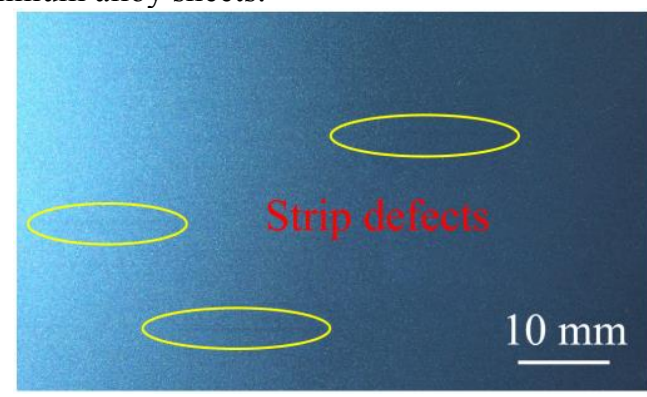

Fig. 2. Anodized 5252 aluminium alloy part of cell phone with strip defects.

Fig.3 showed the morphologies of anodizing film on the anodized AA5252 aluminium alloy part of cell phone. Many round or oval pores were located in the anodizing film. And the porosities of the film in the normal zone and strip zone was $14.4 \%$ and $17.1 \%$, respectively.

Fig.4 showed the relationship between the surface brightness and film porosity, both in normal zone and strip zone of anodized AA5252 aluminium alloy sheets. Compared with the normal zone, the surface brightness of strip zone was lower.

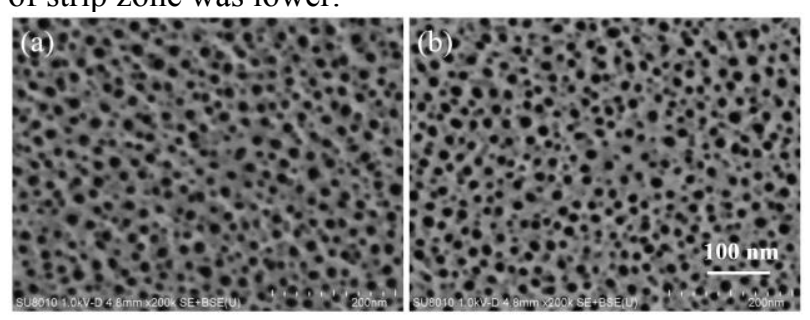

Fig. 3. Anodizing film morphologies of anodized AA5252 aluminium alloy part of cell phone: (a) normal zone with porosity of $14.4 \%$; (b) strip zone with porosity of $17.1 \%$.

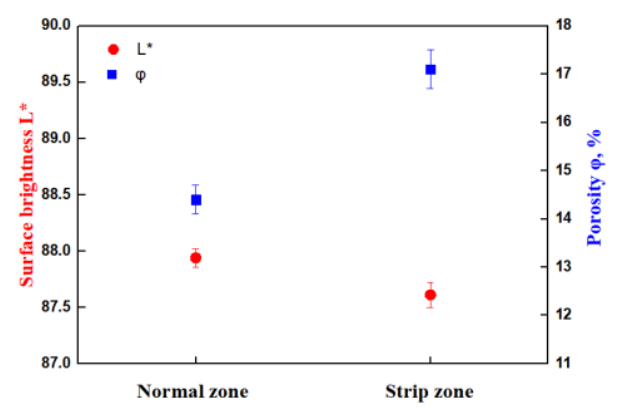

Fig. 4. Surface brightness and film porosity of normal zone and strip zone. 


\subsection{Microstructures}

Fig. 5 showed the distribution of secondary phases in AA5252 aluminium alloys. Several white secondary phases with an equivalent circle diameter less than 1.5 $\mu \mathrm{m}$ located randomly in both the normal zone and strip zone. And they were identified as Fe-rich intermetallic particles by EDS detection. Statistical results showed that the area proportions of the secondary phases in normal zone and strip zone were similar to each other, $0.028 \%$ and $0.022 \%$ respectively. Hence, the effect of secondary phases on the strip defects can be ignored.
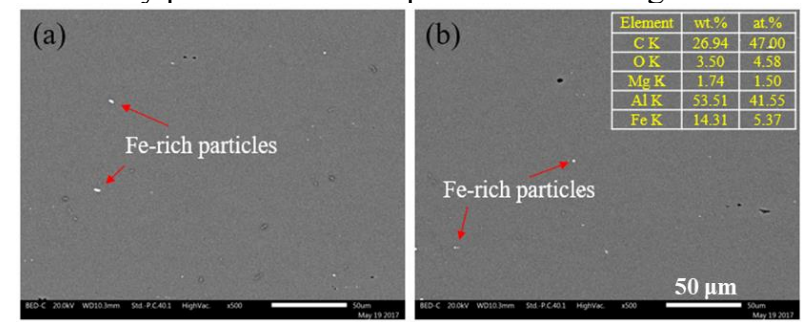

Fig.5. Distribution of secondary phases in AA5252 aluminium alloy sheet: (a) normal zone with area proportion of $0.028 \%$;

(b) strip zone with area proportion of $0.022 \%$.

Fig. 6 showed grain structures in AA5252 aluminium alloy. The equiaxed and elongated grains with large amount of substructures $\left(2^{\circ}<\right.$ misorientation $\left.<15^{\circ}\right)$ distributed in both the normal zone and strip zone. Statistical results showed that the average grain size in normal zone and strip zone was $16 \mu \mathrm{m}$ and $17 \mu \mathrm{m}$, respectively, and the difference between them was not significant.

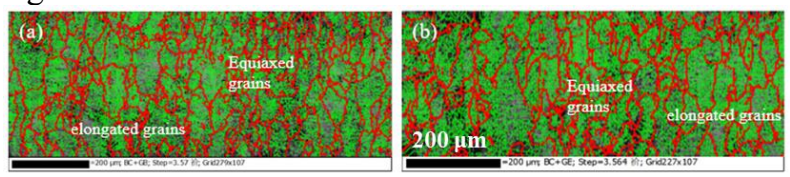

Fig.6. Grain structures in AA5252 aluminium alloy sheet: (a) normal zone with grain size of $16 \mu \mathrm{m}$; (b) strip zone with grain size of $17 \mu \mathrm{m}$.

Fig.7 demonstrated the typical grain orientations in AA5252 aluminium alloys. Table 2 listed the area proportion of typical texture components. Fig.7a and Fig. 7b gave the distribution of typical oriented grains, Copper, S, Brass and Goss oriented grain distributed uniformly along the rolling direction both in the normal zone and strip zone. However, the area proportions of cubic oriented grain in normal zone and strip zone were $11.3 \%$ and 5.6\%, respectively (Fig.7c and Fig.7d), indicating an uneven distributed in AA5252 aluminium alloy sheets.

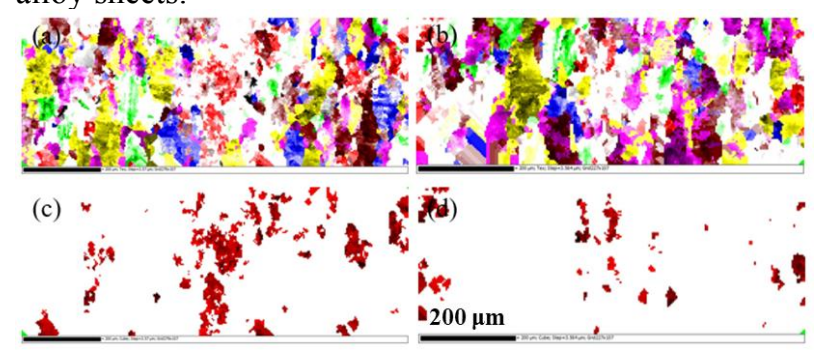

Fig.7. Typical oriented grains in (a) normal zone and (b) strip zone (Blue for Copper, purple for S, yellow for Brass, green for Goss, red for Cube, white for remainder); Cubic oriented grains in (c) normal zone and (d) strip zone.
Table 2. Area proportion of typical grain orientations in

$\left.\begin{array}{lcccccc}\multicolumn{7}{c}{\text { AA5252 aluminium alloy sheets. }} \\ \hline \multirow{2}{*}{\text { ZONE }} & \begin{array}{c}\text { Copper } \\ \{112\}\end{array} & \begin{array}{c}\text { S } \\ \{123\}\end{array} & \begin{array}{c}\text { Brass } \\ \{011\}\end{array} & \begin{array}{c}\text { Goss } \\ \{011\}\end{array} & \begin{array}{c}\text { Cube } \\ \{001\}\end{array} & \begin{array}{c}\text { Remai } \\ \text { nder }\end{array} \\ \hline \multirow{2}{*}{\text { Normal }} & 8.6 & 111> & <634> & <211> & <100> & <100>\end{array}\right)$

\subsection{Characterization of anodizing film in single crystals with different orientations}

Furthermore, in order to confirm the effect of grain orientation on the anodizing film, the single crystal specimens with the (100), (110) to (111) grain orientation were anodized with the same anodizing conditions and studied.

Fig. 8 showed the surface and cross-section morphologies of the anodizing film with different grain orientations. From the top view, the pore size of the anodizing film of (100), (110) and (111) oriented single crystal aluminium was measured as $9.5 \mathrm{~nm}, 8.6 \mathrm{~nm}$ and $8.3 \mathrm{~nm}$, respectively. The porosity was $13.6 \%, 14.7 \%$ and $14.1 \%$, respectively. It can be seen from the crosssection that the angle between the alignment direction of (100), (110) and (111) oriented single crystal anodizing pore and the horizontal direction of aluminium substrate was $90^{\circ}, 76^{\circ}$ and $84^{\circ}$, respectively. And the anodizing film thickness was $10.7 \mu \mathrm{m}, 12.1 \mu \mathrm{m}$ and $12.0 \mu \mathrm{m}$, respectively. Compared with (110) and (111) oriented crystals, the anodizing film on (100) oriented crystal had a lower porosity, and the nanopores seemed more regular. These phenomena were consistent with previous publications ${ }^{[8,9]}$.

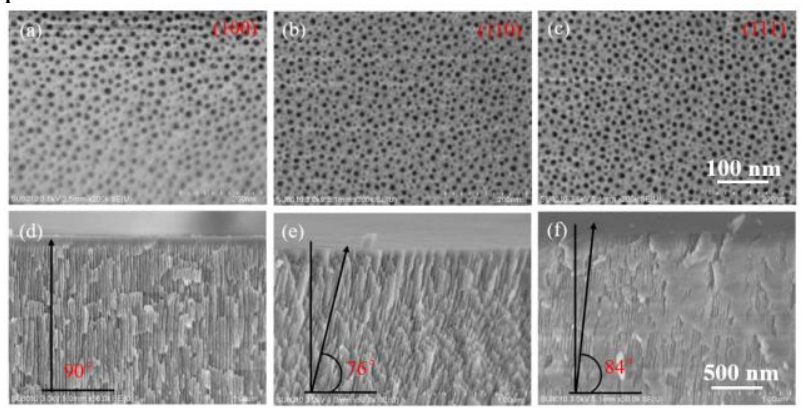

Fig.8. Surficial and cross-section morphologies of anodizing film of single crystals with different grain orientations: (a), (d) 100; (b), (e) 110; (c), (f) 111.

Fig.9 showed the relationship between the surface brightness and porosity of anodized single crystals with different orientations. It can be seen that the surface brightness of anodizing film with (100), (110) and (111) orientation were $93.40,92.71$ and 92.49 , respectively; And the porosity of anodizing film with (100), (110) and (111) orientation were $13.6 \%, 14.7 \%$ and $14.1 \%$, respectively. Compared with (110) and (111) oriented crystals, the higher brightness of anodizing film on (100) oriented crystal had a lower porosity. 


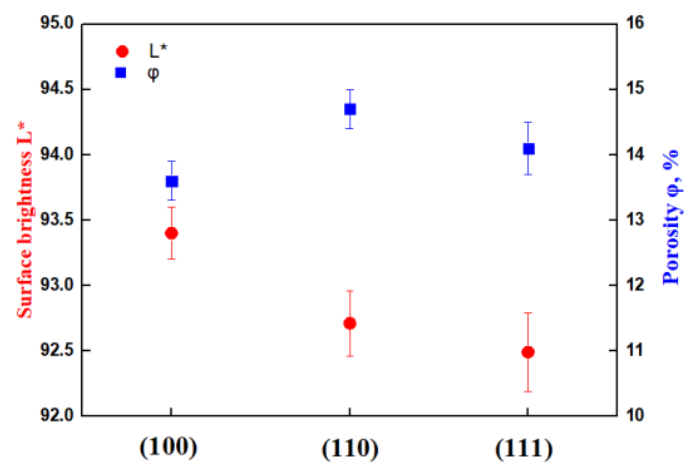

Fig.9. Surface brightness and porosity of anodized single crystal aluminium specimens.

\subsection{Discussion}

Surface brightness of anodized aluminium comes from two mechanisms: the reflected light from anodizing film surface and the reflected light from anodizing film interior. Therefore, the surface brightness was higher, because of the lower porosity and thinner anodizing film in (100) oriented grain, compared with other oriented grains.

Fig.10 gave the sketch maps for the formation mechanism of strip defects in AA5252 sheets. The amount of cubic orientated grains, i.e., (100) oriented grains, in the normal zone was relatively higher than that in strip zone, leading to a lower porosity and a thinner anodizing film. One hand, the lower porosity led to more reflected lights from anodizing film surface, and less proportion of incident lights entered into the pores; On the other hand, the thinner anodizing film reduced the absorption of reflected light in the interior of anodizing pores.

In contrast, the cubic orientated grains appear in lower fraction in the strip zone, leading to a higher porosity and a thicker anodizing film. For the same beam of incident lights, intensity of reflected light from surfaces decreased. Meanwhile, reflected light decreased further due to absorption by the interior of anodizing pores.

To sum up, the formation of strip defects on the surface of anodized AA5252 aluminium alloy sheets was caused by the uneven distribution of cubic orientated grains. The lower proportion of cubic orientated grains in strip zone, leading to dark after anodizing, compared with normal zone with higher proportion of cubic orientated grains.

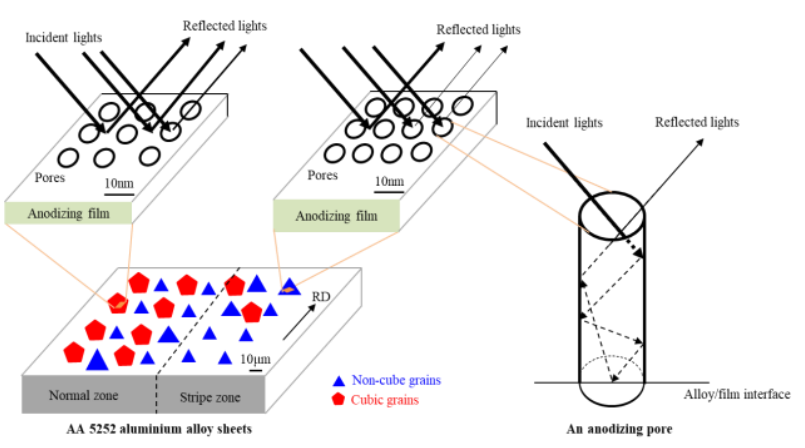

Fig.10. Sketch maps for the formation mechanism of strip defects.

\section{Conclusions}

(1) For the anodized AA5252 aluminium alloy sheet, strip defects zone was darker than normal zone. The strip defects had a higher porosity level in the anodizing film with fewer cubic orientated grains.

(2) The (100) oriented single crystal aluminium after anodizing was brighter than (110) and (111) oriented single crystal aluminium, because its anodizing film is thinner and has lower porosity.

(3) The forming mechanism of strip defects was supposed as uneven distribution of cubic orientated grains. The zones with fewer cubic orientated grains were darker, and were visible as strip defects after anodizing.

\section{References}

1. C.K. Chung, C.H. Tsai, C.R. Hsu, E.H. Kuo, Y. Chen, I.C. Chung. Corros. Sci. 125 (2017) 40-47.

2. A. Nemcova, I. Kubena, M. Smid, H. Habazaki, P. Skeldon, G.E. Thompson. J. Solid State Electr. 20 (4) (2016) 1155-1165.

3. Y. Zhu, S. Wang, Q. Yang, F. Zhou. J. Mater. Eng. Perform. 23 (9) (2014) 3389-3399.

4. L.F. Hu, D.M. Chen, F.R. Shi, S.P. Chen. Surf. Interface Anal. 48 (12) (2016) 1299-1304.

5. J. Evertsson, F. Bertram, L. Rullik, G. Harlow, E. Lundgren. J. Electroanal Chem. 799 (2017) 556-562.

6. Y. Ma, X. Zhou, J. Wang, G.E. Thompson, W. Huang, J.O. Nilsson, M. Gustavsson, A. Crispin. J. Electrochem Soc. 161 (6) (2014) C312-C320.

7. H.L. Zhu, X.Q. Zhang, M.J. Couper, A.K. Dahle. Metall. Mater. Trans. A. 40 (13) (2009) 3264-3275.

8. G. Beck, R. Bretzler. Mater. Chem. Phys. 128 (3) (2011) 383-387.

9. X. Zhou, G.E. Thompson, P. Skeldon. Electrochimi. Acta. 53 (18) (2008) 5684-5691. 